\title{
A NUMERICAL STUDY OF THE RELATIONSHIP BETWEEN THE DIMENSIONLESS PARAMETERS IN THE PROBLEM OF PERIODIC WAVES OF PERMANENT TYPE IN A LIQUID OF FINITE DEPTH*
}

\author{
$\mathrm{BY}$ \\ J. W. TIIOMAS \\ Colorado State University
}

1. Introduction. Consider a periodic wave moving with constant velocity $c$ from right to left on the surface of an inviscid, incompressible fluid which is at rest at infinity. The motion is assumed to be irrotational and two-dimensional. The bottom is horizontal, and the depth of the undisturbed fluid is $h$.

If a constant velocity $c$ from left to right is superposed on the fluid, the wave form is reduced to rest in space, the motion becomes steady, and the velocity at infinity becomes $c$ from left to right. The motion of the fluid contained in one wave length will now be studied.

Levi-Civita has shown in [3] that a wave of invariable form must have a vertical axis of symmetry. Let the $y$-axis be taken vertically to coincide with this axis of symmetry, and let the $x$-axis be in the plane of the undisturbed fluid. The situation is shown in the $z$-plane $(z=x+i y)$ diagram in Fig. 1 . The wave length is denoted by $\lambda$.

Much work has been done toward determining the free streamline for the above wave (see, for instance, [7], [8], and [5], Chapter 14). The wave is determined by two dimensionless parameters $A / h$ and $c^{2} / g h$ where $A$ is the amplitude of the wave and $g$ is the acceleration due to gravity. In [8] Wehausen and Laitone graphed the relationship between $A / h$ and $c^{2} / g h$. (The result is actually a variation of one due to Littman [4].) The graph is obtained by drawing the relationship using both the theories of cnoidal waves and infinitesimal waves. The theory of cnoidal waves is accurate for values of $c^{2} / g h$ near 1 , while the theory of infinitesimal waves should be accurate for values of $A / h$ near 0 . The lines were then smoothed in so as to pass smoothly from one theory to the other. An envelope for this class of curves is then obtained by using results of the highest wave.

In this paper we shall calculate the relationship between $c^{2} / g h$ and $A / h$ by solving the exact problem numerically. We do this by reducing the problem to one of solving a nonlinear integral equation and then use a technique developed by Bueckner [1] to solve this integral cquation. The author used this same approach in [7] to calculate the form of the free streamlines for the cases of finite and infinite depth. The technique is a convergent technique and hence, yields accurate results.

The value of $c$ (as in $c^{2} / g h$ ) is unknown and does not appear in the Milne-Thomson integral equation (as defined in [2]) with which we will be working. For this reason it is

\footnotetext{
* Received July 6, 1973.
} 
necessary to include the reduction of the problem to the integral equation. We do this as briefly as possible in Sec. 2. In Sec. 3 we describe briefly the technique used to solve the integral equation, and hence to calculate points on the graph. Sec. 4 is devoted to a discussion of the results of our calculations.

I would like to thank Joseph B. Keller for suggesting this problem to me.

2. Derivation of the integral equation. As we mentioned above, to be able to calculate the term $c^{2} / g h$ for a given wave profile, it is necessary to have at least a part of the derivation of the integral equation. We shall give a very brief description of this reduction (for more details, see [7]).

We shall map the region occupied by one wave length of the fluid onto a known region in the $Z$-plane $\left(Z=X+i Y=r e^{i t}\right)$. Determining the mapping function will lead to the form of the free surface. Let the known region be $R=\left\{Z: r_{0} \leq r \leq 1\right.$ and $\left.-\pi \leq t \leq \pi\right\}$, where $r_{0}$ is between 0 and $I$ and is yet to be determined. Let the corresponding points in the two planes be denoted by the same letter. Denote this mapping by

$$
z=F(Z) \text {. }
$$

We map the crest $C$ into the point $(1,0)$ in the $Z$-plane and the line $C M$ into the line $C M$ in the $Z$-plane, which we take to lie on the $X$-axis. This function will map $M_{2} T_{2}$ and $M_{1} T_{1}$ into $t=\pi$ and $-\pi$, respectively (sce Fig. 1).

We note that a jump discontinuity is required when going from $T_{2}$ to $T_{1}$. This jump discontinuity can be provided through a term of the form $(i \lambda / 2 \pi) \log Z$, where the remainder of the function can be written in the form of a Laurent expansion in $R$. Thus it is sufficient for $F$ to be of the form

$$
F(Z)=\frac{i \lambda}{2 \pi} \log Z+K_{0}-\sum_{r=2}^{\infty} \frac{a_{n}}{n-1} Z^{1-n}+\sum_{n=0}^{\infty} \frac{a}{n+1} Z^{n+1} .
$$

Let

$$
f(z)=\sum_{n=-\infty}^{\infty} a_{n} z^{n}
$$
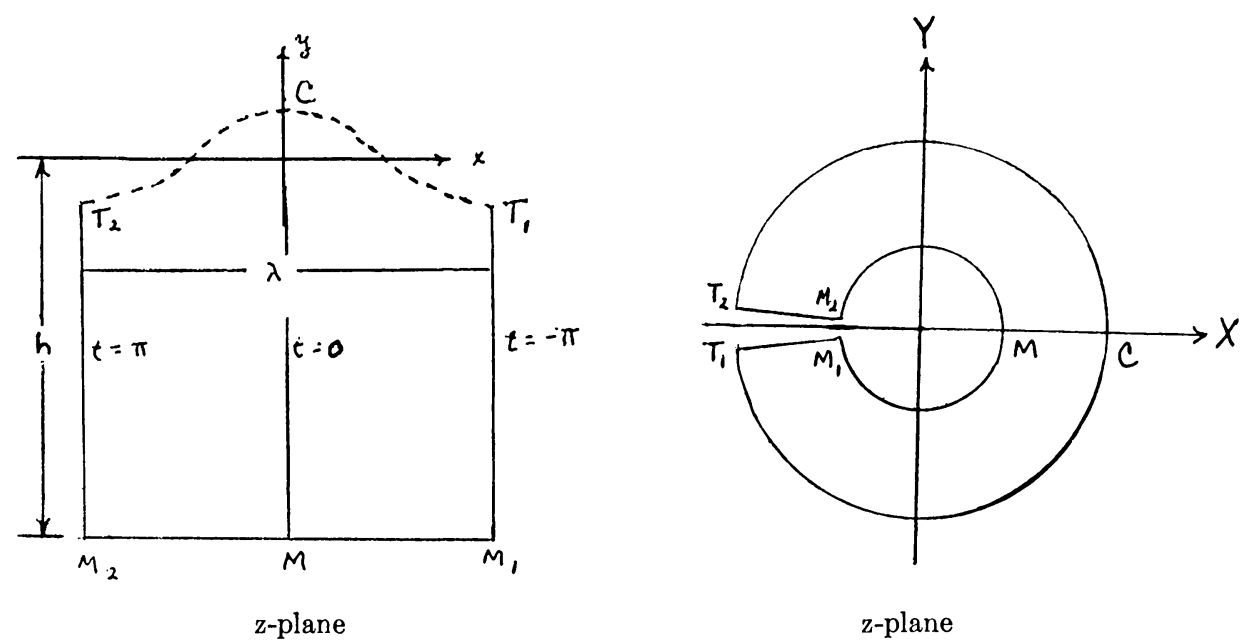

FIG. 1 
in $R$, where $a_{-1}=0$. Hence

$$
\frac{d z}{d Z}=F^{\prime}(Z)=\frac{i \lambda}{2 \pi}\left[\frac{1}{Z}+f(Z)\right] .
$$

The point $Z=r_{0} e^{i t}$ must map into a point on the bottom of the channel so $x-i h=$ $F\left(r_{0} e^{i t}\right)$. In order to have the imaginary part of $F\left(r_{0} e^{i t}\right)$ be the constant $-h$, choose $r_{0}$ so that $K_{0}=0$ and

$$
r_{0}=\exp (-2 \pi h / \lambda)
$$

Thus we can express $F$ as

$$
F(Z)=\frac{i \lambda}{2 \pi}\left[\log Z+\sum_{n=1}^{\infty} \frac{d_{n}}{n}\left(\frac{z^{n}}{r_{0}{ }^{n}}-\frac{r_{0}{ }^{n}}{Z^{n}}\right)\right]
$$

where the $d_{n}$ 's are given in terms of the $a_{n}$ 's and are real.

If we consider the boundary conditions in the $Z$-plane, it is not hard to see that the complex potential

$$
w=-\frac{i c \lambda}{2 \pi} \log Z
$$

satisfies the flow in the $Z$-plane.

Let $Q(r, t) \exp [i \theta(r, t)]$ be the velocity at $(r, t)$, where $Q$ is a real function. Then

$$
-d w / d z=u-i v=Q(r, t) \exp (-i \theta(r, t))=c /(1+Z f(Z)) .
$$

So, if we let

$$
1+Z \digamma(Z)=\exp [T(Z)]
$$

then $T$ can be expressed as

$$
T(Z)=s(r, t)+i \theta(r, t)
$$

where

$$
c \exp (-s(r, t))=Q(r, t) .
$$

Solving (6) for $T$ yields

$$
T(Z) \log (1+Z f(Z))=\sum_{n=-\infty}^{\infty} b_{n} Z^{n},
$$

where the $b_{n}$ are obtainable in terms of the $a_{n}$ of Eq. (2). Since the velocity on the bottom of the channel must be real, $T\left(r_{0} e^{i t}\right)$ must be real, and it is sufficient to let $Z=r e^{i t}$ and write

$$
T\left(r e^{i t}\right)=c_{0}+\sum_{n=1}^{\infty} c_{n}\left(r^{-n} Z^{n}+r^{n} Z^{-n}\right) .
$$

On the surface of the fluid $Z=e^{i t}$, so let $s(1, t)=s(t)$ and $\theta(1, t)=\theta(t)$. Then $\theta$ will give the direction of the tangent along the free streamline and $s$ will give the fluid speed through Eq. (8).

Since the surface is a streamline, Bernoulli's equation with constant pressure, $Q^{2}(1, t)$ $+2 g y=$ constant, must be satisfied, and hence 


$$
(d / d t) Q^{2}=-2 g(d y / d t)
$$

Letting $Z=e^{i t}$, Eqs. (3), (6) and (7) yield

$$
\begin{aligned}
\frac{d z}{d t} & =\frac{i \lambda}{2 \pi} \exp [s(t)+i \theta(t)] \\
& =-\frac{\lambda}{2 \pi} e^{s(t)}[\cos \theta(t)+i \sin \theta(t)] .
\end{aligned}
$$

Equating the real and imaginary parts of Eq. (11) then yields

$$
\begin{aligned}
& d x / d t=-(\lambda / 2 \pi) e^{s(t)} \cos \theta(t), \\
& d y / d t=-(\lambda / 2 \pi) e^{s(t)} \sin \theta(t) .
\end{aligned}
$$

Then Eqs. (8), (10), and (13) yield

$$
\exp (-3 s(t))=\frac{3 g \lambda}{2 \pi c^{2}}\left(\frac{1}{\mu}+\int_{0}^{t} \sin \theta(u) d u\right)
$$

where $1 / \mu$ is the arbitrary constant of integration.

Thus we have established a relationship between $s$ and $\theta$. The next step is to obtain another relationship between $s$ and $\theta$, and hence obtain an equation for just $\theta$. We shall do the next part in a briefer fashion than the preceeding work since all we need from this point on is the resulting integral equation. We shall, however, need Eqs. (12), (13), and (14) obtained above.

The next step is to calculate the coefficients in Eq. (9) in terms of $s$ by considering $T$ on the free surface, i.e. for $r=1$. With a fair amount of manipulation we can write $T$ as

$$
\begin{aligned}
T\left(e^{i t}\right)=\frac{1}{2 \pi} \int_{-\pi}^{\pi} s(x)\left[\frac{1-e^{2 i t}}{1-e^{i t} \cos x+e^{2 i \bar{t}}}\right. & \left.-i \frac{\partial}{\partial x} \log \frac{\sin \frac{1}{\sin 1 / 2}(t+x)}{2(t-x)}\right] d x \\
& +\frac{i}{2 \pi} \int_{-\pi}^{\pi} s(x) \frac{\partial}{\partial x} \log \frac{\operatorname{sn} \frac{K}{\pi}(t+x)}{\operatorname{sn} \frac{K}{\pi}(t-x)} d x
\end{aligned}
$$

where $K$ is the half period of the Jacobi elliptic sine and the last integral is interpreted as a Cauchy principal value. It can easily be shown that the first integral in (15) is zero. Then integration by parts yields

$$
T\left(e^{i t}\right)=\frac{i}{2 \pi}\left[s(x) \log \frac{s n \frac{K}{\pi}(t+x)}{\operatorname{sn} \frac{K}{\pi}(t-x)}\right]_{x=-\pi}^{x=\pi}-\frac{i}{2 \pi} \int_{-\pi}^{\pi} s^{\prime}(x) \log \frac{s n \frac{K}{\pi}(t+x)}{s n \frac{K}{\pi}(t-x)} d x .
$$

But the first term is just $s(t)$, so equating the imaginary parts of Eq. (16) yields

$$
\theta(t)=-\frac{1}{2 \pi} \int_{-\pi}^{\pi} s^{\prime}(x) K(x, t) d x
$$

where $K(x, t)=\log [\operatorname{sn}(K / \pi)(t+x) / \operatorname{sn}(K / \pi)(t-x)]$. This is the desired second relationship between $s$ and $\theta$. Substitution of the $s$ function from Eq. (14) then yields 


$$
\theta(t)=\frac{\mu}{6 \pi} \int_{-\pi}^{\pi} \frac{\sin \theta(x)}{1+\mu \int_{0}^{x} \sin \theta(u) d u} K(x, t) d x .
$$

The symmetry of our functions then allows us to write this as

$$
\theta(t)=\frac{\mu}{3 \pi} \int_{0}^{\pi} \frac{\sin \theta(x)}{1+\mu \int_{0}^{x} \sin \theta(u) g u} K(x, t) d x
$$

The above equation is the Milne-Thomson integral equation that will yield the form of the free streamline for the problem described in the introduction. This was solved by the author in [7] for the eigenvalue $\mu$ and the function $\theta$. (There will be a brief discussion of this method in Sec. 3.) Once we calculate $\mu$ and $\theta$, we use Eqs. (12) and (13) to find the $x$ and $y$ functions (the $y$ function is calculated only up to an additive constant).

Before we solve Eq. (18), we must choose the liquid depth $h$ and wave length $\lambda$. These appear in Eq. (18) indirectly through the half period of the Jacobi elliptic sine $K$. $K$ is determined by the relationship $q=\exp [-4 \pi h / \lambda]$ where $q$ is the nome and equals $\exp \left[-\pi K^{\prime} / K\right]$. Then, after we solve Eq. (18), we have $h$ and we can easily calculate $A$ $(A=|y(\pi)-y(0)|)$. However, we do not have $c$. (Recall that we wish to graph $A / h$ versus $c^{2} / g h$.)

We begin by noting that Eq. (14) yields

$$
e^{s(t)}=\left(\frac{2 \pi c^{2}}{3 g \lambda}\right)^{1 / 3}\left(\frac{1}{u}+\int_{0}^{t} \sin \theta(u) d u\right)^{-1 / 3} .
$$

Eq. (12), along with the fact that $x(0)=0$, yields

$$
x(t)=-\frac{\lambda}{2 \pi} \int_{0}^{t} e^{8(w)} \cos \theta(v) d u .
$$

Then Eqs. (19) and (20) and the fact that $x(\pi)=-\lambda / 2$ give

$$
\frac{c^{2}}{g h}=\frac{3 \lambda \pi^{2}}{2 h}\left[\int_{0}^{\pi}\left(\frac{1}{\mu}+\int_{0}^{\pi} \sin \theta(u) d u\right)^{-1 / 3} \cos \theta(w) d w\right]^{-3}
$$

Thus if $\theta, \lambda, h$, and $\mu$ are all known (and they will be), $c^{2} / g h$ can be determined by Eq. (21).

3. The numerical scheme. We next give a brief discussion of the numerical technique used to calculate the values $A / h$ and $c^{2} / g h$. The central problem was to solve Eq. (18). This is done by letting $u(t)=\mu \sin \theta(t)$ and rewriting Eq. (18) as

$$
u(t)=\sin \left[\frac{1}{3 \pi} \int_{0}^{\pi} \frac{u(x)}{1+\int_{0}^{x} u(y) d y} K(x, t) d x\right]=T(u) .
$$

We then let $u_{0}$ and $\mu_{0}$ denote initial estimates of $u$ and $\mu$, let

$$
\mu_{n}=\frac{\left(u_{n-1}, T\left(u_{n-1}\right)\right)}{\left\|T\left(u_{n-1}\right)\right\|^{2}}
$$

(where (, ) and \| $\|$ are the inner product and norm on $L_{2}$ ), and then define the sequence $\left\{u_{n}\right\}$ by

$$
u_{n}=\mu_{n} T\left(u_{n-1}\right)
$$


Bueckner [1] developed this technique for the problem of calculating the free streamline for the problem discussed in the introduction for a fluid of infinite depth. The author showed in [6] that this technique was applicable also to Eq. (22). In [7] the author gave the results of the solutions to this equation for $h=123.9378$. In this work the author solved Eq. (22) for many $h$ values. Once we have obtained $\theta$ values we can use Eq. (13) to obtain $y$ values (and then $A$ is equal to $|y(0)-y(\pi)|$ ) and Eq. (21) to obtain the value of $c^{2} / g h$.

To obtain the outer envelope of curves given in Fig. 2 we consider the highest wave. The method by which we calculated these values is to let $v(t)=\sin \theta(t)$ and rewrite Eq. (18) as

$$
w(t)=\sin \frac{1}{3 \pi} \int_{0}^{\pi} \frac{w(x:}{\frac{1}{\mu}+\int_{0}^{\pi} w(u) d u} K(x, t) g x=T_{1}(w) .
$$

$\mu$ was then fixed at some large number and we considered the sequence $\left\{w_{n}\right\}$ where

$$
w_{n}(t)=T_{1}\left(w_{n-1}\right) \text {. }
$$

and $w_{0}$ is the initial estimate. At this time there is no convergence proof for the sequence defined in (26), but the numerical results indicate clearly that some sort of theorem is possible.

Again, once we had obtained the $\theta$ values, we were able to proceed as before to calculate $A / h$ and $c^{2} / g h$.

4. Numerical results. The numerical results described above are presented in Table 1 and Fig. 2 below. The table gives the number of the curve (with reference to the graph), the ratio $h / \lambda$ (where we used $\lambda=10 \pi$ ), $A / h$, and $c^{2} / g h$.

Six of the curves ((1)-(6)) in Fig. 2 are the graphs of $A / h$ versus $c^{2} / g h$ for six different values of $h / \lambda$. Curve (7) is the graph of $A / h$ versus $c^{2} / g h$ for the highest wave.

It should be noted that the region in the $(A / h)-\left(c^{2} / g h\right)$ plane that contains the curves is bounded by three curves: curve (7), the curve associated with $h / \lambda=0$, and the line $A / h=0$. Curve (7) is the obvious upper bound for the region since it is associated with the highest wave (and hence the wave with a maximum $A / h$ ) for various choices of $h / \lambda$. The line $A / h=0$ is also another obvious boundary. This line can be thought of being associated with the "flat" wave (no wave) or the case of a fluid of infinite depth. In either case the line is an obvious lower bound for possible values of $A / h$ (surely neither $A$ nor $h$ can be negative). The curve labeled $h / \lambda=0$ shows the relationship between $A / h$ and $c^{2} / g h$ for the solitary wave. It can be thought of as a wave with infinite wavelength. It is clear that the line associated with $h / \lambda=0$ is a boundary of the region (i.e. $h / \lambda$ surely cannot be negative). The curve for $h / \lambda=0$ in Figure 2 is the curve

$$
c^{2} / g h=1+(A / h)-(1 / 10)(A / h)^{2}-(3 / 20)(A / h)^{3}+(9 / 400)(A / h)^{4} .
$$

This is a second-order approximation to the relationship between $c^{2} / g h$ and $A / h$ for the solitary wave. For a discussion of this approximation, see page 711 of [8].

There are several facts that can be used to check the results presented in Fig. 2. In [8] (page 660) it is shown that $c^{2} / g h$ can be expressed in terms of $A$ and $h$ as

$$
c^{2} / g h=\frac{1}{m h} \tanh m h\left[1+A^{2} m^{2} \frac{8+\cosh 4 m h}{8 \sin ^{4} m h}+\cdots\right]
$$


TABLE 1.

\begin{tabular}{|c|c|c|c|c|c|c|}
\hline Number & (1) & (1) & (1) & (1) & & \\
\hline$h / \lambda$ & 0.6 & 0.6 & 0.6 & 0.6 & & \\
\hline$A \mid h$ & 0.005185 & 0.10763 & 0.14663 & 0.20252 & & \\
\hline$c^{2} \mid g h$ & 0.265005 & 0.27643 & 0.28649 & 0.30699 & & \\
\hline Number & (2) & (2) & $(2)$ & (2) & & \\
\hline$h / \lambda$ & 0.3 & 0.3 & 0.3 & 0.3 & & \\
\hline$A \mid h$ & 0.01899 & 0.24156 & 0.27919 & 0.35717 & & \\
\hline$c^{2} \mid g h$ & 0.50702 & 0.53863 & 0.555023 & 0.57781 & & \\
\hline Number & (3) & (3) & (3) & (3) & & \\
\hline$h / \lambda$ & 0.2 & 0.2 & 0.2 & 0.2 & & \\
\hline$A \mid h$ & 0.01326 & 0.31778 & 0.36557 & 0.50237 & & \\
\hline$c^{2} \mid g h$ & 0.67778 & 0.72578 & 0.74129 & 0.79721 & & \\
\hline Number & (4) & (4) & (4) & (4) & & \\
\hline$h / \lambda$ & 0.15 & 0.15 & 0.15 & 0.15 & & \\
\hline$A \mid h$ & 0.02984 & 0.25816 & 0.36794 & 0.54365 & & \\
\hline$c^{2} \mid g h$ & 0.78474 & 0.81952 & 0.85394 & 0.92950 & & \\
\hline Number & (5) & $(5)$ & $(5)$ & $(5)$ & (5) & (5) \\
\hline$h / \lambda$ & 0.13655 & $0.136 \tilde{5})$ & 0.13655 & 0.13655 & 0.13655 & 0.13655 \\
\hline$A \mid h$ & 0.01547 & 0.10796 & 0.30932 & 0.37796 & 0.46207 & 0.51817 \\
\hline$c^{2} \mid g h$ & 0.813 .51 & 0.82062 & 0.86689 & 0.89129 & 0.92640 & 0.95260 \\
\hline Number & (6) & (6) & (6) & $(6)$ & (6) & \\
\hline$h / \lambda$ & 0.10632 & 0.10632 & 0.10632 & 0.10632 & 0.10632 & 0.10632 \\
\hline$A \mid h$ & 0.01676 & 0.29091 & 0.39328 & 0.43636 & 0.52072 & \\
\hline$c^{2} \mid g h$ & 0.88130 & 0.94167 & 0.98415 & 1.0043 & 1.04674 & \\
\hline Number & (7) & (7) & (7) & (7) & (7) & \\
\hline$h / \lambda$ & 0.6 & 0.39789 & 0.3 & 0.24926 & 0.21709 & \\
\hline$A \mid h$ & 0.23327 & 0.344477 & 0.44240 & 0.50540 & 0.55230 & \\
\hline$c^{2} \mid g h$ & 0.34140 & 0.50250 & 0.65345 & 0.75077 & 0.82900 & \\
\hline Number & (7) & (7) & (7) & (7) & (7) & \\
\hline$h / \lambda$ & 0.2 & 0.18366 & 0.15 & 0.13655 & 0.10632 & \\
\hline$A \mid h$ & 0.58037 & 0.60289 & $0.6598 i$ & 0.67433 & 0.71924 & \\
\hline$c^{2} \mid g h$ & 0.88053 & 0.92039 & 1.03836 & 1.07376 & 1.19664 & \\
\hline
\end{tabular}

Thus for $A / h=0$ we see that

$$
c^{2} / g h=(1 / m h) \tanh (m h) .
$$

Thus for $h=6 \pi, 3 \pi, 2 \pi, 1.5 \pi, 4.29$ and 3.34 , we see that $c^{2} / g h=0.2650,0.5066,0.6772$, $.7810,0.8098$, and 0.8737 , respectively. These points are also plotted on the graph in Fig. 2. It should be noted that these points agree very nicely with the curves drawn by the numerical data.

Also in [8] (page 712) we see by the theory of cnoidal waves that the ratio of the maximum amplitude to $h(A / h)$ for the highest wave when $k=1$ (where $k$ is the modulus and is such that $k^{2}=m$ ) should equal 0.7143 . This too is plotted on the graph and also agrees with our numerical results.

The results represented in Fig. 2 agree closely with the results given in [8] for small $A / h$ and $c^{2} / g h$ near 1 (as they well should). Because of the method used to draw the 


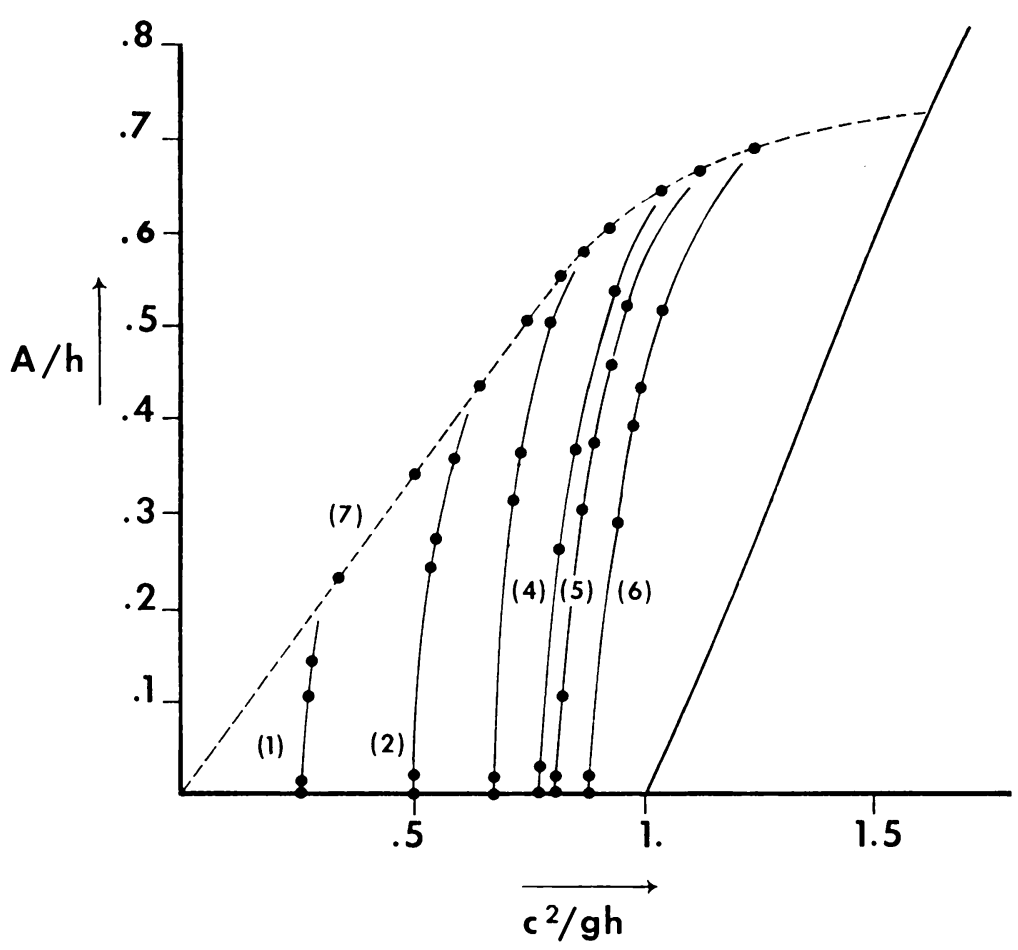

FIG. 2. The graphs of $A / h$ versus $c^{2} / g h$ for six different constant values of $h / \lambda$ and of the limiting case of $A / h$ versus $c^{2} / g h$ for the highest wave. The numerical values are given in Table 1 .

graphs in [8], we maintain that our graphs will be more reliable for the values "between" shallow water theory and cnoidal wave theory.

There are no rate of convergence proofs for the numerical schemes used here. The results indicate, however, the most convergence acted much like a geometric sequence with ratio $1 / 2$. This observation was made by observing many (all of the points that are plotted and more) applications of the iterative scheme.

\section{REFERENCES}

[1] IIans F. Bueckner, An iterative method for solving nonlinear integral equations, MRC Tech. Report \#207, University of Wisconsin, 1960

[2] W. E. Conway and J. W. Thomas, Free streamline problems and the Milne-Thomas integral equations, J. Math. Phys. Sci. VIII, 67-92 (1974)

[3] T. Levi-Civita, Determination rigoureuse des ondes permanentes d'ampleur finie, Math. Ann. 93, 264$314(1925)$

[4] Walter Littman, On the existence of periodic waves near critical speed, Comm. Pure Appl. Math. 10, 243-269 (19:57)

[5] L. M. Milne-Thomson, Theoretical hydrodynamics, ith edition, New York, Macmillan Company, 1968

[6] J. W. Thomas, On the exact form of gravity waves on the surface of an inviscid liquid, dissertation, University of Arizona, 1967

[7] J. W. Thomas, Irrotational gravity waves of finite height: a numerical study, Mathematica 15, 139-148 (1968)

[8] John V. Wehausen and Edmund V. Laitone, Surface waves, in Encyclopedia of physics IX , 446-814 $(1960)$ 